\title{
COMPARISON OF THE EFFECT OF LASER THERAPY AND THERAPEUTIC ULTRASOUND IN THE MANAGEMENT OF CHRONIC OSTEOARTHRITIC KNEE PAIN: A RANDOMISED CONTROLLED TRIAL.
}

\author{
Journal website at; \\ http://mrtbjournal.org/index.php/njmr/issue/current/showToc \\ ${ }^{1}$ S HANIF, ${ }^{1}$ AR SALIM, ${ }^{2}$ S LAMINA, ${ }^{1}$ UL ISA \\ ${ }^{1}$ Department of Physiotherapy Faculty of Medicine Bayero University, Kano \& \\ ${ }^{2}$ Biomedical Technology Dept., School of Health Technology, \\ Federal University of Technology, Owerri. \\ Correspondence to: \\ Lamina Sikiru \\ siklam_86@yahoo.co.uk \\ Tel: 08025294087
}

\section{SUMMARY}

Objective: This randomized controlled clinical trial was designed to evaluate and compare the therapeutic efficacy of Low Laser Therapy (LLLT) and Therapeutic Ultrasound (TUS) in the symptomatic management of knee osteoarthritis.

Methods: Twenty two subjects with chronic osteoarthritis of the knee were recruited, aged-matched and grouped into 3 (control, LLLT \& TUS) groups. The control group received 18 sessions of standardized protocol care of exercise therapy (low intensity for 15 minutes and 3 times per week for 6 weeks). The LLLT and TUS groups, in addition to the standardized exercise therapy protocol received 18 LLLT (Laser class 3b, frequency of $5 \mathrm{KHz}$, wave length of $810 \mathrm{~nm})$ treatments for 10 minutes and 18 TUS sessions (continuous, intensity: $0.5 \mathrm{~W} / \mathrm{cm} 2$, frequency: $1 \mathrm{MHz}$ ) for 12 minutes respectively. Visual analogue scale (VAS) was used to measure knee pain and knee joint range of motion (ROM) was also assessed. Data was analyzed using ANOVA test to determine groups' significant difference.

Results: Findings of the study revealed no significant contribution of LLLT and TUS over control (exercise) in VAS and ROM at $p<0.05$.

Conclusions: The study affirms and concluded that exercise therapy should form the basis and bulk adjunct physical therapy in the management of osteoarthritis over Laser therapy and TUS.

Key words: Laser; Ultrasound; Exercise; Physiotherapy.

\section{INTRODUCTION}

Osteoarthritis (OA) is the clinical and pathological outcome of a range of disorders that result in structural and functional failure of synovial joints. It has been considered as diseases of articular cartilage, but the current concept holds that OA involves the entire joint organs including the subchondral bone, menisci, ligaments, periarticular capsule and synovium (Martins et al., 2001), as such, it is a major cause of locomotor pain, the single most important rheumatological cause of disability and handicap, and an important health care challenge with major resource implications (Badley \& Tennnnant, 1992).

Physiotherapy modalities commonly used in the management of OA include interferential therapy, Transcutaneus Electrical Nerve Stimulator (TENS), Therapeutic Ultrasound (TUS), Low Level Laser Therapy (LLLT), Acupuncture, exercise therapy and orthotic devices (Bjordal et al.,2007; Ottawa Panel, 2004; Deyle, 2000). Until recently OA was generally considered to be an irreversible consequence of ageing: however, researches in the last decade has led to the view that $\mathrm{OA}$ is an active disease with a potential for treatment (Aldred \& Key, 2003), but still no agent has been proven to be curative in its treatment. The knee joint is the commonly affected joint with pain, reduced range of motion (ROM), crepitus and joint swelling as its common impairment (Altman, Abadie \& Avovae, 2001).

TUS has been suggested for alleviating pain and inducing increase in range of motion in acute peri-articular conditions (Downing \& Weinstein, 1993), but it is still not clear whether ultrasound can be used to reduce the symptoms of OA (Robertson, Backer \& Duuck, 1993). Early 
clinical trials attempting to examine the effectiveness of therapeutic ultrasound were methodologically flawed (Nussbaum, 1997). Holmes and Rudland (1991) reported that out of the 18 clinical trials they evaluated, most had methodological flaws including lack of control groups, correct criteria of statistical analysis of the result and lack of standardized treatment. As with the ultrasound, LLLT elicits a number of biological effects in the tissue (Young \& Dyson, 1991). Review of clinical evidence indicates that LLLT could potentially be effective in relieving pain associated with chronic OA; however, several laboratories are currently working to elucidate the mechanism behind LLLT (Bjordal et al., 2006).

The Ottawa Panel (2004) evidence suggested that LLLT could be applied without addition of other modalities to solve pain related problems of arthritis. However, its effectiveness is still controversial (Brosseau et al., 2005). Also, research has shown that LLLT can be used to effectively treat many conditions stimulating body's natural repair process using the healing nature of light. However, there is still a need for further investigation on its clinical effectiveness and other electrotherapy modalities, of which ultrasound appears to have effects on biological process at cellular level just like LLLT. For the purpose of this study, 2 null hypotheses were formulated and tested:

(1) There would be no significant difference between LLLT group and control group in pain and ROM.

(2). There would be no significant difference between TUS group and control group in pain and ROM.

\section{MATERIALSANDMETHODS}

Study design: A double blind randomized independent group design was used in data collection. Clinical trial was approved by the management and ethical committees of the various hospitals were subjects were recruited.

Population and subjects: Thirty two subjects were recruited from the population of 88 patients with knee OA attending Aminu Kano Teaching Hospital (AKTH), Murtala Muhammad Specialist Hospital (MMSH), and National Orthopedic Hospital Dala (NOHD) Kano, Nigeria.

Sample Size: Thirty two (32) ambulatory chronic (> 3 months) knee OA patients were recruited, according to the American College of Rheumatology (ACR)(American College of Rheumatology, 2000) criteria.

Inclusion criteria: Knee pain for more than 25 of the past 30 days, morning stiffness of less than 30 minutes, crepitus in the knee or knee pain for more than 25 of the past 30 days and Osteophytes on X-ray examination of the knee (Altman et al., 1986). Subjects with knee OA, who had pain for more than 25 of the past 30 days and at least two of the following three criteria, were also recruited; Erythrocyte Sedimentation Rate (ESR) $<20 \mathrm{~mm} / 1$ st hour, osteophytes on X-ray examination, or obliteration of joint space.

Exclusion criteria: Subjects with low back pain, metallic implants at the lower limb, peripheral vascular disease, tumor in the lower limb, patients on cholesterol-lowering drugs, patients with solar eczema photosensitivity and sunlight hypersensitivity and patients on immunosuppressant. Subjects with respiratory, hepatic or renal failure, history of drug abuse, severe illnesses and arthroplasty of the knee joint.

Subjects that met the inclusion criteria's were recruited, aged matched and randomly assigned to 3 groups: control group, TUS group and LLLT group.

Data collection procedure: Ethical approval was sought for, in the various hospitals were subjects are recruited. Written informed consent was obtained from the subjects after they were brief about the study. Only participants who gave their consent were included in the study. At the first appointment, each subject's age, stature, weight and body mass index (BMI) were determined using standardized protocols and procedures (International Society for the Advancement of Kinanthropometry, 2001). Pretest washout: A pretest washout period of 7 days was required prior to data collection; The purpose of the wash out period was to get rid of all forms of analgesics previously taken,

Pretest (pretreatment) assessment: Subjects degree of stiffness (range of motion [ROM]) and pain perception were assessed using Goniometer (Model G300, Manufactured by Whitehall Manufacturing Hydrotherapy and Health Care Products) and visual analogue scale (VAS)(Hoof, 2007) respectively. These assessments were performed on the first and the last intervention days (pre and post treatment).

Treatment protocol for control group: All the subjects in this group received 18 sessions of therapeutic exercise. The exercise program consist of 5 minutes warm-up stretching program followed by range of motion exercise and finally isometric exercise and isotonic exercise the exercise were localized to the quadriceps and hamstrings group of muscles. The exercise was of low intensity for 15 minutes. The exercise protocol used was adopted from Deyle and Henderson (2000).

Treatment protocol for TUS group: Subjects in this group received 18 sessions of therapeutic ultrasound (Model 27335 manufactured by Chattanooga Group, a division of encore Medical, England) on the lateral and medial side of the knee, at a frequency of $1 \mathrm{MHz}$ and $3 \mathrm{MHz}$, intensity of $0.5 \mathrm{~W} / \mathrm{cm} 2$ for 12 minutes over a period of six weeks, all necessary precautions, preparation of both apparatus and patient were carried out appropriately prior to treatment. Subjects were assessed for any condition contraindicating the use of therapeutic ultrasound. Following ultrasonic therapy, subjects in this group also received therapeutic exercise as in control group (Robertson et al., 2001; Speed, 2001).

Treatment protocol for LLLT group: This group received 18 sessions of LLLT, over a period of six weeks; all necessary precautions, preparation of both apparatus and subjects were carried out prior to treatment. Subjects were assessed appropriately for any condition contraindicating the use of LLLT. LLLT was applied according to 
manufacturers (THOR LX international) recommendation as follows:

Time: duration of 2 minute was used per spot size with total of 10 minute

Treatment area: painful areas chosen from medial and lateral side of the knee Type

of laser: Class 3B

Frequency: $5 \mathrm{kHz}$ was used

Wave length: all treatment was carried out at same wave length of 810nm, 100mw (Bjordal et al., 2006; Brosseau et al., 2005; Bulow et al., 1994). Following ultrasonic therapy, subjects in this group also received therapeutic exercise as in control group.

Posttest washout: Following treatment and prior to post treatment data collection, another 5 days wash bout period was also established.

Posttest assessment: ROM and VAS were administered to assess stiffness and pain respectively as earlier described in the pretest procedure. The purpose of the posttest was to assess the effect of treatment (outcome variables).

All pre and post test measurements were recorded on a data sheet. Twenty two subjects (6 from control, 6 from TUS and 10 from LLLT groups) completed the six weeks treatment program.

\section{Data analysis procedure}

The data was analyzed using descriptive statistics of means, standard deviation. Inferential statistics of analysis of variance (ANOVA) was used to compare groups' physical, baseline characteristics and outcome variables. In the outcome ANOVA, the differences between the pretest and posttest values (changed score) were used as the dependent variables. All statistical analyses were performed on an IBM compatible microcomputer using the Statistical Package for the Social Sciences (SPSS) (Windows version 16.0 Chicago IL, USA), and a probability level of 0.05 was used to indicate significance.

\section{RESULTS}

Twenty two subjects with knee OA(12 females \& 10 males) aged between 47 and 83 years were included in the study, subjects' mean \pm SD was $62.86 \pm 11.36$. Table 1 indicates ANOVA, groups' physical and baseline characteristics. Table 2; ANOVA summary indicated no group significant difference in all the parameters assessed at $\mathrm{p}<0.05$. Figure $1 \& 2$ showed pre-posttest and changed score values for pain (VAS) and ROM respectively.

\section{DISCUSSION}

Table 1. Groups physical and base line characteristics (ANOVA[N=22])

\begin{tabular}{lllll}
\hline Variables & $\begin{array}{l}\text { Control } \\
\mathbf{X} \pm \text { SD } \\
\mathbf{n = 6}\end{array}$ & $\begin{array}{l}\text { LLLT } \\
\mathbf{X} \pm \text { SD } \\
\mathbf{n = 1 0}\end{array}$ & $\begin{array}{l}\text { TUS } \\
\mathbf{X} \pm \text { SD } \\
\mathbf{n = 6}\end{array}$ & $\begin{array}{l}\mathbf{p - ~} \\
\text { values }\end{array}$ \\
\hline Age(year) & $59.17 \pm 12.64$ & $64.70 \pm 11.27$ & $63.50 \pm 11.38$ & 0.655 \\
Height(meter) & $1.59 \pm 0.04$ & $1.60 \pm 0.06$ & $1.62 \pm 0.06$ & 0.493 \\
Weight(kg) & $68.17 \pm 5.67$ & $72.60 \pm 5.32$ & $75.17 \pm 6.55$ & 0.129 \\
BMI(kg/m2) & $27.04 \pm 1.38$ & $28.60 \pm 3.41$ & $28.70 \pm 3.85$ & 0.580 \\
Pain & $7.50 \pm 1.05$ & $6.30 \pm 1.34$ & $7.83 \pm 1.19$ & 0.050 \\
ROM(degree) & $118.17 \pm 2.48$ & $117.70 \pm 3.89$ & $117.50 \pm 3.78$ & 0.945 \\
\hline
\end{tabular}

*significant, $\mathrm{p}<0.05$

Table 2: Comparison of groups changed score variables (ANOVA summary [ $\mathrm{N}=22]$ )

\begin{tabular}{lll}
\hline Variables & F-values & p-values \\
\hline VAS & 3.158 & .065 \\
ROM & 1.095 & .355 \\
\hline
\end{tabular}

*significant $\mathrm{p}<0.05$

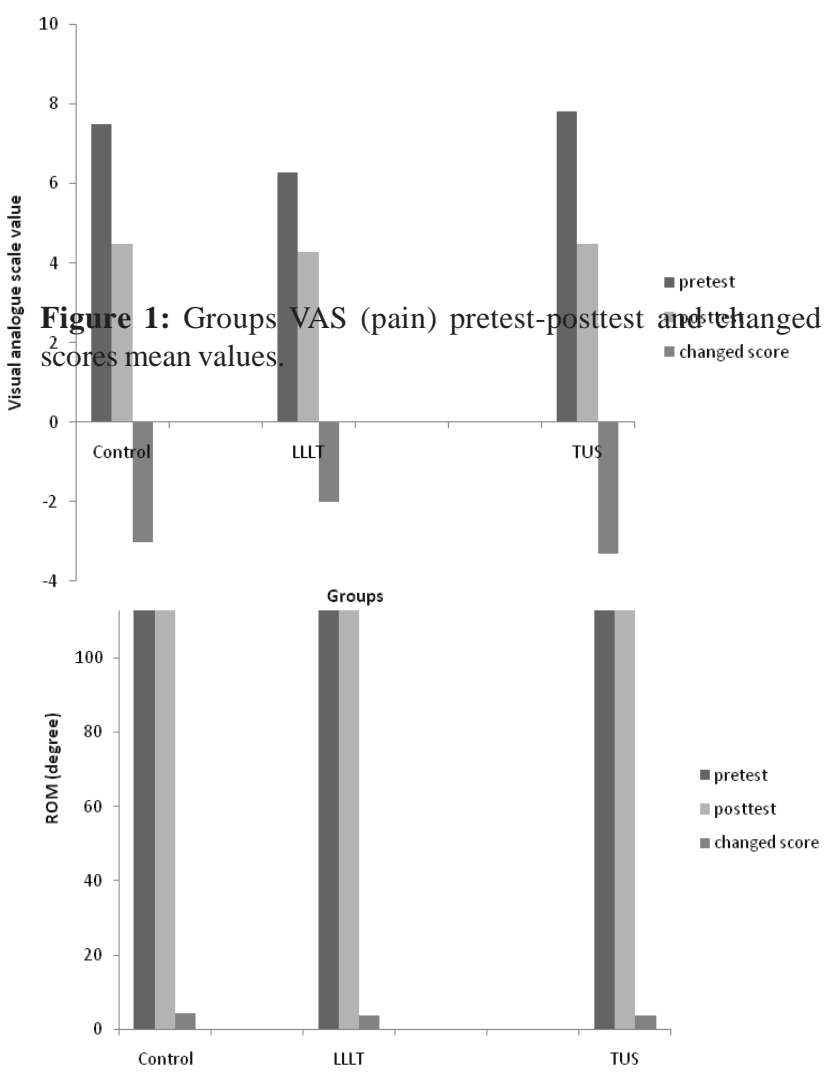

Figure 2: Groups ROM fremtest-posttest and changed scores mean values 
The purpose of this study was to test the null hypotheses that; there would be no significant difference between LLLT and TUS groups over control group in pain perception and ROM. Both null hypotheses were supported by the results of the present study. The outcome of the study revealed no significant difference between the 3 groups after 18 treatment $(6$ weeks) session of LLLT(LLLT \& exercise), TUS (TUS \& exercise) and control (exercise).on the contrary, exercise has been shown to improved function, decreased pain and stiffness, and increased walking distance in six minutes compared to placebo treatment of ultrasound therapy at a sub therapeutic intensity (Thomas, 2000); also Huang \& Lin (2003) reported that there is strong evidence for the benefits of exercise in relieving pain and improving functional status in patients with knee OA, after muscle strengthening program.

The use of LLLT in knee OA has been subject to limited studies, the results of which are conflicting. Result of the present study is in agreement with the findings of Bulow, Danneskiold-Samsoe \& Danneskiold-Samsoe (1994) the outcome of which indicated no significant difference in pain and functional ability after 4 treatment sessions per week compared to the control group. Basimia, Sadeghipoor and Esmeeeli-Djavid (2001) used IR diode laser $810 \mathrm{~nm}$ once per day for 5 consecutive days, followed by a two days interval, the treatment was for 2 minutes per tender point, and the outcome indicated significant difference in pain relief and quality of life in $70 \%$ of the patients $(\mathrm{P}<0.05)$ with no significant change in $\mathrm{ROM}$ of the patients. Even though the authors used same wave length as the present study, difference in patients' physical characteristics, duration of the treatment and type of the laser diode used might explain the difference between the study of Basimia et al, (2001) and the present study.

Bjordal et al (2007) concluded that a 4-week intensive treatment regimen of LLLT for knee OA offer clinically relevant short term pain relief, with the effect persisting for at least 4 weeks after treatment. However, this finding is in contrary to the present study, this difference in findings might be attributed to the different in laser modality, as well as treatment parameter selected in both study. In disparity to this findings, Tascioglu et al (2004) used Ga-As laser with power output of $50 \mathrm{~mW}$ and $83 \mathrm{~nm}$ wave length to treat knee OA at painful sites 5 times a week for a period of 2 weeks, the result indicated no significant difference at all time (3 weeks and 6 month after treatment) compared to baseline

Therapeutic ultrasound is one of the several physiotherapy modalities suggested for the management of pain and reduced functional ability due to OA. The result of this study is in disagreement with report of Srbly (2008) who concluded that ultrasound had beneficial effects in knee OA following a systemic review of 17 trials that 12 studies consistently reported that ultrasound has therapeutically beneficial effects on pain and functional outcomes of the patients. Another similar review study was conducted by Gam and Johannsen (1996) they reviewed articles published between 1950 and 1992 on ultrasound used to treat patients with musculoskeletal problems, OA inclusive; they concluded that only 22 of the 293 articles were methodologically adequate and that, any contribution of ultrasound to the then treatment outcome was not evident on the basis of the finding of the control trials.

Robinson, Baker \& Duck (2004) also found no evidence that TUS is more effective than placebo ultrasound in the management of People with OA. Speed (2001) reported that no evidence was found for pain relief with TUS treatment, even though the significant improvement seen in the TUS group might be due to the effect of exercise. the most likely reason why the present study vary from that of the findings of Srbly (2008) may be attributed to methodological differences, differences in patients physical characteristics, type of modalities and the selected doses, since the optimal doses range of TUS for many conditions are yet to be established, even though findings of many previous studies and this trial revealed no significant difference in the efficacy of TUS.

Different laser and TUS may have different effectiveness in different stages of knee OA and treatment parameters, particularly the wave length, energy density, mode and duration of the therapy (Basford, 1998). Therefore, the failure of 18 treatment sessions of both LLLT and TUS to show significant precedence might be due to the modality, dosages and wave length used in the study, and on the other hand patients' variation with other studies might be a reason. Based on the findings of the present study, it is thereby affirmed and recommended that exercise therapy should form the basis and bulk adjunct physical-therapy in the management of osteoarthritis over Laser therapy and TUS.

While our findings provide a rational base for recommending therapeutic exercise as the basis and bulk adjunct physical modality in the symptomatic management of OA over LLLT and TUS, there are some limitations of the study including few participants and non sham LLLT and TUS control. These factors warrant more attention in future studies.

\section{REFERENCES}

- Aldred H, Key JI (2003): Degenerative joint disease. Athritis Rheuamatology 22: 481-3.

- Altman R A, Bloch D, Bole G, Boreinstein D, Brandt, K (1986): Development of criteria for the classification and reporting of OA. Arthritis Rheumatology 29: 1039-1049.

- Altman RD, Abadie E, Avouac B (2001): Group for Respect of, and S. Ethics in, Total joint replacement of hip or knee as an outcome measure for structure modifying trials in osteoarthritis. Osteoarthritis \& Cartilage 13(1): 13-19.

- American College of Rheumatology (2000): Recommendations for the medical management of 
osteoarthritis of the hip and knee: update. American College of Rheumatology Subcommittee on Osteoarthritis Guidelines. Arthritis Rheum. 43(9): 19051915

- Badley EM, Tennant A. (1992): Changing profile of joint disorders with age: findings from a postal survey of the population of Calderdale, West Yorkshire, United Kingdom. Annual Rheumatology Disease. 51: 366-71.

- Basford JR (1998): Physical agents. In: DeLisa JA, Gans BM (eds.) .Rehabilitation Medicine: Principles and Practice, Third edition. Lippincott Raven Publishers: Philadelphia.

- Basimia A, Sadeghipoor G, Esmaeeli-Djavid G (2001): The Effect of LLLT on OA of the Knee. Training and resources for clinical Excellence in Energetic Therapies. www.HealingLightSeminars.com.

- Bjordal JM, Bogen B, Lopes-Martins RA, Johnson MI, Bogen B Chow R, Ljunggren A (2007): E.Short-term efficacy of physical interventions in osteoarthritic knee pain. A systematic review and meta-analysis of randomised placebocontrolled trials. BMC Musculoskelet Disord 8: 51-55

- Bjordal JM, Johnson MI, Iversen VV, Aimbire F, R.A.B. LM. (2006): Low Level Laser Therapy (LLLT) in acute pain. A systematic review of possible mechanisms of action and clinical effects in randomized placebo-controlled trials . Photomed Laser Surg.24:158168

- Brosseau L, Welch V, Wells G, Gam A, Harman K, Morin M, Shea B, Tugwell P (2005): LLLT (class I, II \& III)for the treatment of OA. Photomed Laser Surg 23(3): 453-8

- Bulow PM, Danneskiold-Samsøe J, Danneskiold-Samsøe B (1994): Low power GaAlAs laser treatment of painful osteoarthritis of the knee. A double-blind controlled study. Scandinavian Journal of Rehabilitation and Medicine 26:155159.

- Deyle G.D, Henderson N.E, Matekel R.L, Ryder M.G, Garber M.B, Allison S.C. (2000): Effectiveness of manual physical therapy and exercise in osteoarthritis of the knee: A randomized, controlled trial Ann Intern Med. 132:173-81

- Deyle GD (2000): Effectiveness of manual physical therapy and exercise in osteoarthritis of the knee. A randomized, controlled trial. Ann Intern Med 132: 173-181.

- Downing DS, Weinstein A (1993): Ultrasound therapy of subacromial bursitis. A double blind trial. Phys Ther 66:1949.

- Gam AN, Johannson F (1996): Ultrasound therapy in musculoskeletal disorders: a meta-analysis. Pain 63:8591
- Holmes MAM, Rudland JR (1991): Clinical trials of ultrasound treatment in soft tissue injury: a review and critique. Physiotherapy Theory and Practice 7:163175.

- Hoof, k (2007): Pelvic inflammatory disease. Ther Umsch 64(7): 3658 .

- Huang MA, Lin YS (2003): A comparison of variousn therapeutic exercise on the functional status of patients with OA. Semin arthritis Rheumatology 36: 398-406

- International Society for the Advancement of Kinanthropometry (2001): International standards for anthropometric assessment. Patche Fstroom, South Africa: ISAK.

- Martins B, Knobloch M, Tschopp A, Jucker A, Howald H (2001): Is excessive running predictive of degenerative hip disease? Controlled study of former elite athletes. Bio Medical Journal 299: 91-3

- Nussbaum EL (1997): Ultrasound: to heat or not to heatthat is the question. Physical Therapy Review 2:5972

- Ottawa Panel. (2004): Ottawa Panel Evidence-Based Clinical Practice Guidelines for Electrotherapy and Thermotherapy Interventions in the Management of Rheumatoid Arthritis in Adults. Phys Ther 84(11): 1016-43.

- Robertson VJ, Baker KG, Duck FA. (2001): A review of therapeutic ultrasound: biophysical effects. Phys Ther. 81:13511358.

- Speed CA (2001): Therapeutic ultrasound in soft tissue lesions. Rheumatology 40: 1331- 1336

- Srbely JZ (2008): Ultrasound in the management of osteoarthritis: part 1: a review of the

- Tascioglu JK, Armagon H, Tabak F, Coraopci I, Oner I (2004): Ga-As laser in the management of Knee OA. Elsevier print.

- Thomas KS, Doherty MH (2002): Home based exerciceprogramme for kneepain and knee OA:Randomized control trial. Biomedical Journal 325:752-756

- Young SR, Dyson M (1991): Effects of therapeutic ultrasound on the healing of full-thickness exercised skin lesion ultrasonic. World laser therapy association 28: 175 180 . 